\title{
JAK2 and Endothelial Function: New Options for Anti-Thrombotic Therapies
}

\author{
Thomas Kietzmann ${ }^{1}$ \\ ${ }^{1}$ Faculty of Biochemistry and Molecular Medicine, Biocenter Oulu, \\ University of Oulu, Oulu, Finland
}

Thromb Haemost 2018;118:1512-1514.

The activation of Janus kinases (JAKs) is a crucial enzymatic step in the signal transduction of many growth factors and cytokines. Four members, JAK1, JAK2, JAK3 and tyrosine kinase 2 (TYK2) have been identified and they all are wellknown due to their function in the haematopoietic and immune system. Their profound involvement in the regulation of the immune response made JAKs to be attractive drug targets for inflammatory disorders such as rheumatoid arthritis ${ }^{1}$ and cancers of the immune system such as multiple myeloma. ${ }^{2}$ Genetic alterations of JAKs were described for all four JAKs and associated with human diseases. While inherited loss of function mutations in JAK3 and TYK2 were found in severe combined immune deficiency and atopic dermatitis, respectively, somatic gain of function mutations in JAK1, JAK2 and JAK3 resulted in myeloproliferative neoplasms (MPNs) and leukaemia/lymphomas. For example, generation of fusion proteins consisting of JAK2 and TEL, breakpoint cluster region $(\mathrm{BCR})$ or pericentriolar material-1 were described in chronic myeloid leukaemia, acute myeloid leukaemia or acute lymphoblastic leukaemia., ${ }^{3,4}$ In addition, the point mutation JAK2V617F gained fame after it was identified as the major driving force in non-BCR-ABL1 MPNs in particular in polycythaemia vera, essential thrombocythaemia and primary myelofibrosis. ${ }^{5-7}$ The JAK2V617F mutation can also be found, but much less frequent, in the hypereosinophilic syndrome, chronic or juvenile myelomonocytic leukaemia, acute myeloid leukaemia and refractory anaemia with ringed sideroblasts (for review see Haan et al). ${ }^{8}$

So far, it was common belief that the acquired somatic JAK2V617F mutation affects mainly haematopoietic stem cells, multi-potent progenitor cells, ${ }^{9,10}$ cells from the lymphoid lineage ${ }^{11,12}$ and some differentiated cells like granulocytes. ${ }^{7}$ However, several recent studies reported occurrence of the JAK2V617F mutation in endothelial cells (ECs) of patients with MPN and Budd-Chiari syndrome. ${ }^{13-15}$ In fact, thromboembolic events represent a major cause of morbidity and mortality during the chronic phase of MPN
Address for correspondence Thomas Kietzmann, MD, PhD, Faculty of Biochemistry and Molecular Medicine, Biocenter Oulu, University of Oulu, Aapistie 7, Fl-90220 Oulu, Finland (e-mail: tkietzm@gwdg.de).

patients, before the onset of the accelerated phase with evolution to myelofibrosis or acute leukaemia. Thrombotic manifestations in MPN patients are affecting unusual sites like the portal vein or splanchnic veins. Indeed, splanchnic vein thrombosis affects 0.9 to $5 \%$ of the patients with polycythaemia vera and 3 to $10 \%$ of the patients with essential thrombocythaemia. ${ }^{16}$ Vice versa, approximately 40 and 30\% of Budd-Chiari syndrome or portal vein thrombosis patients, respectively, have underlying MPNs. ${ }^{17}$

Although the thrombotic risk in the MPN patients can be associated with an increased haematocrit, leucocytosis or platelet dysfunction, it is so far unknown what makes MPN patients to be prone to thromboembolic events. Since ECs are critically involved in the regulation of vascular structure, cellular adhesion, vascular tone and thromboresistance, it may be very well possible that the JAK2V617F mutation in ECs of an MPN patient pre-disposes him to thrombosis.

Indeed, this problem was tackled by Guadall et al and the outcome of their studies is described in an article of the current issue of Thrombosis and Haemostasis. ${ }^{18}$ To address this problem, the authors used isogenic JAK2V617F and JAK2 wild-type (WT) induced pluripotent stem (iPS) cells from an MPN patient and redirected these iPS cells towards the endothelial lineage. Tube formation assays in matrigel and nitric oxide formation as characteristic parameters of ECs first revealed that the iPS cells can be differentiated towards the endothelial lineage and that no principal difference between the cells from the two genotypes was detectable. However, the authors detected a gain of function in JAK2V617F cells when compared with the WT cells; this became visible by increased levels of phosphorylated JAK2 and signal transducer and activator of transcription 3 (STAT3). These findings resemble the enhanced intra-cellular JAK2/STAT3 signalling as observed in JAK2V617F haematopoietic cells. Moreover, the authors observed an increased proliferation in the cells with the JAK2V617F mutation when compared with WT ECs. (c) 2018 Georg Thieme Verlag KG Stuttgart · New York
DOI https://doi.org/ $10.1055 / \mathrm{s}-0038-1668586$. ISSN 0340-6245. 
Next, the authors were able to link the observed findings to an activated status of ECs that is prone to thrombotic events by looking at the formation of Weibel-Palade bodies and expression of von Willebrand factor (VWF) and P-selectin (CD62P). All these parameters, numbers and fluorescence intensity of Weibel-Palade bodies as well as expression of vWF and P-selectin were significantly higher and also accompanied by accumulation of P-selectin at the cell surface in JAK2V617F cells when compared with WT cells.

To characterize the consequences of JAK2V617F expression in the ECs further, the authors obtained the respective transcriptomic profile of these cells. The authors found 428 genes being differentially expressed (259 up- and 169 downregulated) in JAK2V617F versus WT cells. Importantly, a gene set enrichment analysis showed that various over-expressed genes were highly related to pro-inflammatory and proadhesive properties, to extracellular matrix regulation, and to generation of glycoproteins, processes that are also involved in venous stenosis and thrombosis. In particular, the induction of a pro-thrombotic phenotype of the JAK2V617F cells was underlined by the over-expression of the interleukin (IL)-33 receptor; IL-33 is known to induce tissue factor expression in ECs. ${ }^{19}$ This was further supported by the dysregulation of SULF1 expression, which is involved in desulphation of cellular heparan sulphate proteoglycans, and of the sphingosine-1-phosphate receptor 3 that contributes to vWF release from Weibel-Palade bodies as well as to P-selectin surface exposure. ${ }^{20,21}$

Next, the authors functionally substantiated the prothrombotic expression profile and examined whether the interactions between white blood cells (WBCs) and the JAK2V617F ECs or WT ECs is showing a difference. To this end, the authors established cell adhesion assays using WBC from healthy donors or from MPN patients. The authors found that JAK2V617F cells bound stronger to WBC from healthy donors than their WT counterparts. This difference was even more exaggerated upon usage of WBCs from a JAK2V617F MPN patient. Together, these assays confirmed that the JAK2V617F mutation provides a pro-adherent phenotype to ECs.

Altogether, the novelty aspect in the study of Guadall et al consists in the overarching finding that differentiation of pluripotent stem cells from MPN patients harbouring the JAK2V617F mutation could give rise to ECs which display pro-adherent and pro-thrombotic features, which can contribute to the thrombotic events seen in MPN patients. Moreover, the findings also support the view that JAK2V617F ECs present in the bone marrow niche could contribute to the development of myelofibrosis.

Overall, and with respect to thrombosis, this needs to be seen together with the other cells critically involved in blood clotting and thrombus formation such as platelets. Apart from the erythropoiesis, the JAK2 V617F point mutation in the normal haematopoietic progenitor cells causes also the growth of megakaryocytes, which results in higher production of platelets. Although platelets lack the nucleus to direct ribonucleic acid transcription, they and even more their megakaryocytic progenitors react very well to stimulation by JAK2 activating factors such as thrombopoietin, or
IL-3. ${ }^{22,23}$ A study investigating platelet haemostatic and adhesive molecules found that platelets from essential thrombocythaemia JAK2V617F patients expressed higher membrane tissue factor and P-selectin. ${ }^{24}$ In addition, a recent report indicated that JAK2 can be involved in collagen-induced platelet activation. ${ }^{25}$ Hence, presence of the gain of function JAK2V617F mutation in megakaryocytes and platelets and as shown here for ECs explains, at least in part, the high frequency of major arterial and venous thrombotic complications as well as the micro-vascular, platelet-mediated disturbances in MPN patients. Consequently, this raises the thought about the druggability of the thrombotic status by JAK2 inhibitors. In fact, only one inhibitor, ruxolitinib, targeting JAK2/JAK1 has been approved for treatment of hydroxyurea-intolerant polycythaemia vera, and intermediate- or high-risk myelofibrosis but not essential thrombocythaemia. ${ }^{26}$ Another inhibitor, tofacitinib, targeting preferentially JAK1 and JAK3 has been approved for methotrexate-resistant rheumatoid arthritis. ${ }^{27}$ Ruxolitinib is a well-tolerated drug, which decreases inflammatory cytokines, leukocytosis and thrombocytosis and thereby improves the clinical status as well as prolongs survival of patients with myelofibrosis. $^{26,28,29}$ Although it is common believe that the ruxolitinib effects are mainly caused by the inhibition of cytokine action, it will be interesting to see its effects in other MPNs such as essential thrombocythaemia where it is in latephase clinical trials. Further, since JAK2 activation is mainly triggered by IL-3, IL-5, granulocyte-macrophage colonystimulating factor, interferon- $\gamma$, erythropoietin, thrombopoietin, growth hormone and prolactin, ${ }^{30}$ one may, in addition to MPNs and leukaemia, think about mountain sickness, acromegaly, prolactinomes or inoperable hypophyseal tumours as treatment entities. Given the relative safety of ruxolitinib, and in light of the findings with ECs from the paper of Guadall et al, it is provoking to speculate that JAK2 inhibition, either with ruxolitinib or other more specific new inhibitors such as pacritinib or NS-018, could represent an avenue for more wider therapeutic options associated with EC proliferation, for example, in atherosclerosis or myocardial infarction. This would even not require a JAK2 gain of function mutation since ruxolitinib inhibits both, mutated and WT JAK2. Overall, more research, more testing and more clinical trials are needed to improve the current picture.

Conflict of Interest

None.

\section{References}

1 Riese RJ, Krishnaswami S, Kremer J. Inhibition of JAK kinases in patients with rheumatoid arthritis: scientific rationale and clinical outcomes. Best Pract Res Clin Rheumatol 2010;24(04): 513-526

2 Mahindra A, Cirstea D, Raje N. Novel therapeutic targets for multiple myeloma. Future Oncol 2010;6(03):407-418

3 Lacronique V, Boureux A, Valle VD, et al. ATEL-JAK2 fusion protein with constitutive kinase activity in human leukemia. Science 1997;278(5341):1309-1312 
4 Griesinger F, Hennig H, Hillmer F, et al. A BCR-JAK2 fusion gene as the result of a $\mathrm{t}(9 ; 22)(\mathrm{p} 24 ; \mathrm{q} 11.2)$ translocation in a patient with a clinically typical chronic myeloid leukemia. Genes Chromosomes Cancer 2005;44(03):329-333

5 Zhao R, Xing S, Li Z, et al. Identification of an acquired JAK2 mutation in polycythemia vera. J Biol Chem 2005;280(24): 22788-22792

6 James C, Ugo V, Le Couédic JP, et al. A unique clonal JAK2 mutation leading to constitutive signalling causes polycythaemia vera. Nature 2005;434(7037):1144-1148

7 Levine RL, Wadleigh M, Cools J, et al. Activating mutation in the tyrosine kinase JAK2 in polycythemia vera, essential thrombocythemia, and myeloid metaplasia with myelofibrosis. Cancer Cell 2005;7(04):387-397

8 Haan C, Behrmann I, Haan S. Perspectives for the use of structural information and chemical genetics to develop inhibitors of Janus kinases. J Cell Mol Med 2010;14(03):504-527

9 Baxter EJ, Scott LM, Campbell PJ, et al; Cancer Genome Project. Acquired mutation of the tyrosine kinase JAK2 in human myeloproliferative disorders. Lancet 2005;365(9464):1054-1061

10 Jamieson CH, Gotlib J, Durocher JA, et al. The JAK2 V617F mutation occurs in hematopoietic stem cells in polycythemia vera and predisposes toward erythroid differentiation. Proc Natl Acad Sci U S A 2006;103(16):6224-6229

11 Bellanné-Chantelot C, Chaumarel I, Labopin M, et al. Genetic and clinical implications of the Val617Phe JAK2 mutation in 72 families with myeloproliferative disorders. Blood 2006;108(01): 346-352

12 Delhommeau F, Dupont S, Tonetti C, et al. Evidence that the JAK2 G1849T (V617F) mutation occurs in a lymphomyeloid progenitor in polycythemia vera and idiopathic myelofibrosis. Blood 2007; 109(01):71-77

13 Sozer S, Fiel MI, Schiano T, Xu M, Mascarenhas J, Hoffman R. The presence of JAK2V617F mutation in the liver endothelial cells of patients with Budd-Chiari syndrome. Blood 2009;113(21): 5246-5249

14 Rosti V, Villani L, Riboni R, et al; Associazione Italiana per la Ricerca sul Cancro Gruppo Italiano Malattie Mieloproliferative (AGIMM) investigators. Spleen endothelial cells from patients with myelofibrosis harbor the JAK2V617F mutation. Blood 2013;121(02):360-368

15 Teofili L, Martini M, Iachininoto MG, et al. Endothelial progenitor cells are clonal and exhibit the JAK2(V617F) mutation in a subset of thrombotic patients with Ph-negative myeloproliferative neoplasms. Blood 2011;117(09):2700-2707

16 De Stefano V, Qi X, Betti S, Rossi E. Splanchnic vein thrombosis and myeloproliferative neoplasms: molecular-driven diagnosis and long-term treatment. Thromb Haemost 2016;115(02): 240-249

17 Kiladjian JJ, Cervantes F, Leebeek FW, et al. The impact of JAK2 and MPL mutations on diagnosis and prognosis of splanchnic vein thrombosis: a report on 241 cases. Blood 2008;111(10): 4922-4929

18 Guadall A, Lesteven E, Letort G, et al. Endothelial cells harbouring the JAK2V617F mutation display pro-adherent and pro-thrombotic features. Thromb Haemost 2018;118(09). Doi: 10.1055/s0038-1667015

19 Stojkovic S, Kaun C, Basilio J, et al. Tissue factor is induced by interleukin-33 in human endothelial cells: a new link between coagulation and inflammation. Sci Rep 2016;6:25171

20 Nussbaum C, Bannenberg S, Keul P, et al. Sphingosine-1-phosphate receptor 3 promotes leukocyte rolling by mobilizing endothelial P-selectin. Nat Commun 2015;6:6416

21 van Hooren KW, Spijkers LJ, van Breevoort D, et al. Sphingosine-1phosphate receptor 3 mediates sphingosine-1-phosphate induced release of Weibel-Palade bodies from endothelial cells. PLoS One 2014;9(03):e91346

22 Nurden AT, Viallard JF, Nurden P. New-generation drugs that stimulate platelet production in chronic immune thrombocytopenic purpura. Lancet 2009;373(9674):1562-1569

23 Imura Y, Stassen JM, Vreys I, Lesaffre E, Gold HK, Collen D. Synergistic antithrombotic properties of G4120, a RGD-containing synthetic peptide, and argatroban, a synthetic thrombin inhibitor, in a hamster femoral vein platelet-rich thrombosis model. Thromb Haemost 1992;68(03):336-340

24 Tefferi A, Elliott M. Thrombosis in myeloproliferative disorders: prevalence, prognostic factors, and the role of leukocytes and JAK2V617F. Semin Thromb Hemost 2007;33(04):313-320

25 Lu WJ, Lin KC, Huang SY, et al. Role of a Janus kinase 2-dependent signaling pathway in platelet activation. Thromb Res 2014;133 (06):1088-1096

26 Bose P, Verstovsek S. JAK2 inhibitors for myeloproliferative neoplasms: what is next? Blood 2017;130(02):115-125

27 Vannucchi AM, Harrison CN. Emerging treatments for classical myeloproliferative neoplasms. Blood 2017;129(06):693-703

28 Harrison C, Kiladjian JJ, Al-Ali HK, et al. JAK inhibition with ruxolitinib versus best available therapy for myelofibrosis. $\mathrm{N}$ Engl J Med 2012;366(09):787-798

29 Gäbler K, Behrmann I, Haan C. JAK2 mutants (e.g., JAK2V617F) and their importance as drug targets in myeloproliferative neoplasms. JAK-STAT 2013;2(03):e25025

30 Babon JJ, Lucet IS, Murphy JM, Nicola NA, Varghese LN. The molecular regulation of Janus kinase (JAK) activation. Biochem J 2014;462(01):1-13 